\title{
Cardiac CT Angiography Manual
}

\section{R. Pelberg and W. Mazur}

New York, NY: Springer, 2007, 142 pages, $\$ 49.95$

Cardiac CT angiography is a rapidly growing imaging modality that provides vital diagnostic information, and the technique is changing continuously as a result of new research and further clinical experience. Therefore, interest in training for this new modality is increasing exponentially, but few practical books dedicated to the topic are available to educate cardiologists and radiologists who are in training or in practice.

This small handbook is intended to be a helpful summary of the field of cardiac CT angiography and calcium scoring that may aid in teaching of the technique. Consequently, difficult concepts are simplified, and figures are depicted for the purpose of clarity and easy understanding.

The authors collected and compiled information from the peer-reviewed literature and also from their personal experience in performing this technique. They believe the information in this book to be reliable and in accord with current standards. They also designed this handy book for trainees in cardiology and radiology by making difficult-to-understand concepts easy to comprehend with the aid of intuitive diagrams. Terminology and concepts are thoroughly defined and explained.

This book is organized into 2 sections: Section I, with 2 chapters, introduces calcium scoring and covers its essentials. Section II, with 6 chapters, provides an overview of cardiac CT angiography and covers its indications, concepts, essentials, reports, and applications. This book is clearly written, with figures of good quality and updated references. It admirably accomplishes its goal of serving as an educational tool and reference for cardiologists and radiologists.

E. Edmund Kim M.D. Anderson Cancer Center Houston, Texas 